\title{
Dry Sand Erosion Damage Characteristic of Fibers Induced by Solid Particle Impact
}

\author{
Limin BAO*, Yasuyuki Sato*, Danna QIAN*, Satoshi Simakawa*, \\ Zhijuan Pan** and Shigeru Hinata* \\ * Faculty of Textile Science and Technology, Shinshu University \\ 3-15-1 Tokida, Ueda, 386-8567, Japan \\ **School of Material Engineering, Soochow University \\ 178 Ganjiangdonglu Suzhou, Jiangsu 215021, China
}

\begin{abstract}
Bag filters made of fibrous components are widely used for cleaning exhaust gas. Filter damage caused by dust collection results from the erosion of the filter during particle collision. We developed an experiment and evaluation method to investigate the erosion characteristics of fiber. Using the suggested kinetic energy of impact, we evaluated the erosion wear characteristics of fibers under different experiment conditions. The results demonstrated that the erosion wear of fiber depended on the kinetic energy of impact. The erosion wear level changes based on the material characteristics (brittle or ductile) and on the impact angle of solid particles. In addition, the erosion characteristics are greatly influenced by strength and elongation of materials.
\end{abstract}

KEY WORDS: Erosion, Solid Particle Impact, Wear, Fiber, Experiment and Estimate of Erosion.

\section{INTRODUCTION}

Currently, bag filters of fibrous components are widely used for cleaning exhaust gas in municipal refuse incinerator plants and industrial waste facilities. These components are compressed fibers in a weakly bound random array.

Bag filters have the advantage of efficiently eliminating dioxins that are absorbed in particulate substances and environmental pollutants from exhaust gas. However, the continued use of fiber components creates damage, leading to dangerous gas leaks in the immediate area of the incineration facility. Therefore, it is necessary to determine the effective life of the bag filter.

We researched many damaged bag filters in a refuse incinerator plant ${ }^{1}$. Two patterns were observed: fiber fractured by tension, and fiber fractured by wear. Most $(92.8 \%)$ of the observed fiber was fractured by wear. Filter damage by dust collection results from the erosion of the filter caused by particle collision. As illustrated in Fig. 1, grinding particles and solid surface projection when particles crush against material causes a minor cutting wear effect. This is dry sand erosion wear.

Since dioxin contamination is a major concern, it is necessary to predict the wear life so that a bag filter may be designed that prevents the destruction of fiber. The erosion mechanism of fibrous material must be clarified.

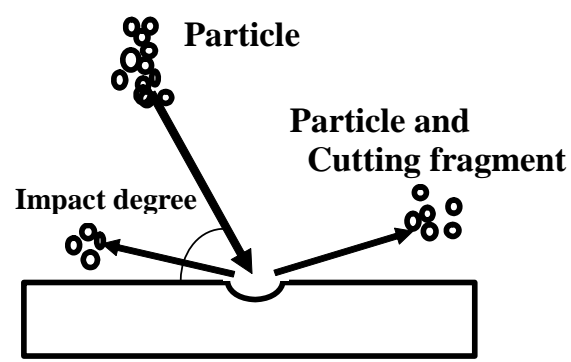

Material

Fig. 1 Schema of erosion

Schick suggested methods to measure the wear characteristics of fiber and yarns ${ }^{2}$. He examined the mechanism and factors affecting the wear of fibers and yarns and incorporated the results into his application. However, he did not clarify the erosion properties of fibers.

Others, however, have reported on the erosion wear of a materials. Hashimoto ${ }^{3}$, Kimura ${ }^{4}$ and others studied wear resistance in terms of impact speed, impact angle and particle density for many materials. These research activities contributed to the development and application of the materials. However, their studies focused mainly on metals and inorganic materials.

Since the $1980 \mathrm{~s}^{5)}$, the investigation of the erosion behavior of materials extended from metals to polymers and their composites. One matrix that has been eroded includes epoxy, polypropylene and polyethylene, which were listed by A.P. Harsha and co-workers ${ }^{6}$. To reinforce this, researchers changed the type of fiber to fiberglass reinforced plastic (FRP). To investigate the mechanism of erosion wear, the majority of experiments were carried out under various impact angles, speeds and shapes of the impact particle. The wear resistance of polymer composites that contain reinforcement fiber is usually lower than that of neat polymers ${ }^{7-8}$.

The erosion of the fiber materials has not been studied. Therefore, there are many unknowns in this field.

In our research, we attempted to develop a suitable device and experiment method to measure the erosion damage of fibrous materials induced by the impact of solid particles. Using a prototype apparatus, we simulated fibrous erosion. The eroded elements were investigated to determine the fiber erosion mechanism.

\section{EXPERIMENT}

Measurement Apparatus and Method

To determine the wear characteristics of metals and materials under given conditions, the difference in weight is determined before and after the test. The difference is defined as the amount of wear, which is used to estimate the 
characteristics of wear resistance of materials.

Fibrous materials are extremely difficult to evaluate using the same method as for metals. Fibrous materials are lighter than metal materials, so the amount of wear is very small. Additionally, the particles migrate into fibrous cavities. Therefore, the difference in weight of fibrous material measured is not determined precisely, and the measurement errors are large. A new experimental method is needed to determine the amount of wear of fibrous (fiber and yarn) materials.

Our proposed experiment procedure is described below.

Fig. 2 presents a schematic drawing of the test system for determining erosion wear of fibers.

(1) As illustrated in Fig. 2, the fiber is fixed with both ends of the load measurement equipment and an electrical slider. We ensured that the fiber did not come loose in this test.

(2) A solid particle is shot from nozzle of an air gun into the fibrous materials to create fiber wear.

(3) After a specified time, the electric slider subjects the fibrous materials damaged by experiment to a tensile test. The breaking load of the fibrous materials is measured by load cell. We compare the fiber breaking load before and after wear to evaluate the fiber's wear level.

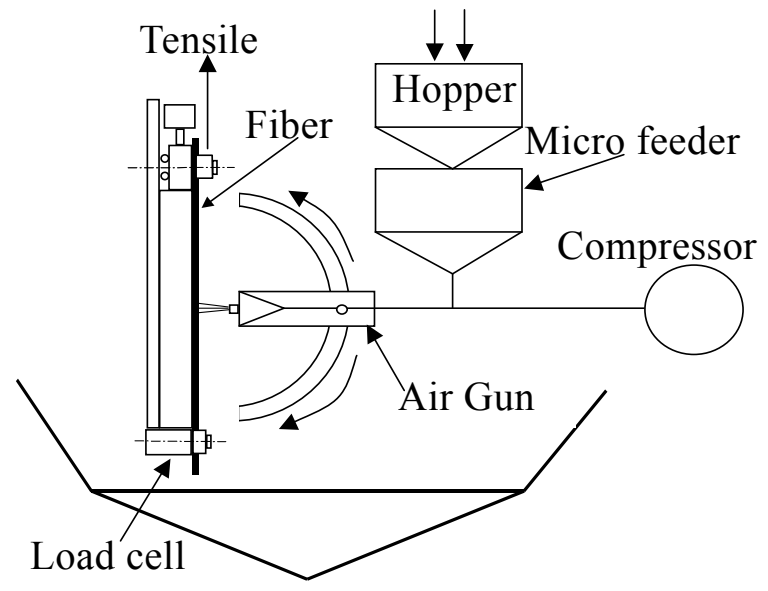

Fig. 2 Schematic drawing of the test system for evaluating fiber's wear.

The breaking load is measured by load cell (LUB-B 100N, KYOWA Co. Ltd.) and a sensor interface board (PCD-100, KYOWA Co. Ltd.). The electric slider moves at 0.5 millimeter per second during the tensile test. The solid particle is saved by a hopper, and is provided at constant quantity by micro feeder (ME-1, Tsutsui Rikagaku Kikai Co., Ltd.). The compressed air was created from an air compressor. The solid particle velocity is regulated by the air pressure in the compressor. The number and velocity of solid particle are controlled by wear resistance of the fibers. The distance between the air gum nozzle and fiber is $10 \mathrm{~mm}$; nozzle diameter is $6 \mathrm{~mm}$. Impact angles are set to six stages (90, 75, 60, 45, 30 and 15 degrees), and tests are performed at each stage.

To maintain a constant supply of solid particles, humidity was set to $65 \%$ and temperature was set to 20 degrees Celsius.

\section{Sample Materials}

Fiber specimen properties are presented in Table 1. Procon is PPS (Polyphenylen Sulfide) fiber developed by Toyobo Co. Ltd. Procon has excellent chemical resistance against acids and alkaline media at high temperatures of 150 to 190 degrees Celsius. Procon has a trilobal cross section and increases fiber surface to provide good filtration efficiency; it is therefore, is suitable for bag filters. DE-75 is a glass filament from Unitika Glass Fiber Co., Ltd.. Kurebara135d is a polyester filament form Shimasensyoku Co., Ltd.. They are also used as bag filters. However, DE -75 has excellent high-temperature properties and chemical resistance. The experiment was repeated five times.

Considering the fly ash in refuse incinerator plant, the white alumina polishing material from Showa Denko K.K. was used for the particles. It means diameter is 11.5 micrometer, and the standard deviation is 21.5 .

Table 1 Details of samples

\begin{tabular}{|c|c|c|c|}
\hline $\begin{array}{c}\text { Sample } \\
\text { name }\end{array}$ & Material & $\begin{array}{c}\text { Diameter of } \\
\text { fiber }(\mu \mathrm{m})\end{array}$ & $\begin{array}{c}\text { Yarn counts } \\
(\mathrm{dtex})\end{array}$ \\
\hline Procon & PPS & 20 & 250 \\
\hline Kurebara135d & Polyester & 20 & 135 \\
\hline DE-75 & Glass & 6 & 675 \\
\hline
\end{tabular}

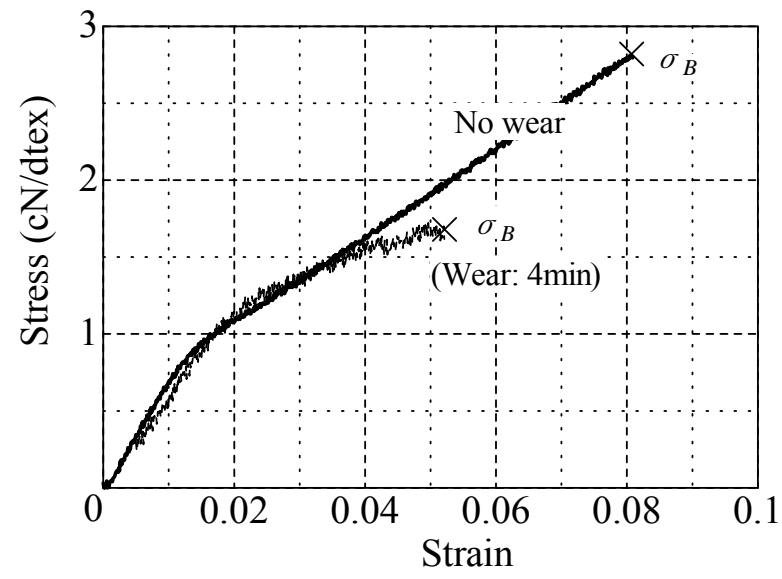

Fig.3 Stress-strain curve of fibers before and after wear. (Procon, Impact angle 90 degrees)

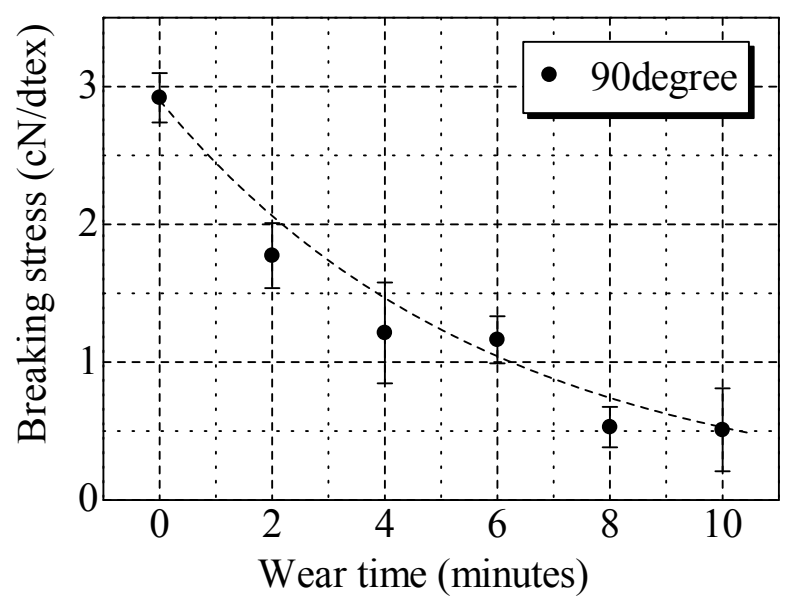

Fig.4 Breaking stress-wear time curve of fibers. (Procon, Impact angle 90 degrees) 


\section{RESULTS AND DISCUSSION}

Using the device depicted in Fig. 2, we investigated the erosion of fiber at a various impact angles and wear times. Fig. 3 plots a stress and strain curve of fiber before and after wear. Impact angle is 90 degrees, and sample is Procon (in table). The particle supply rate was $0.816 \mathrm{~g} / \mathrm{min}$, and collision speed was $80.14 \mathrm{~m} / \mathrm{sec}$. The maximum stress of the curve was defined as the breaking stress. The breaking stress after wear was less than that before wear.

Figure 4 illustrates the relationship between wear time and breaking stress of the Procon fiber. The experiment conditions are the same as in Fig. 3. The breaking stress depended on the wear time. A long wear time decreases the fiber breaking stress. The amount of decrease represents the material erosion characteristic.

The contact area of fiber and particle is changed by changing the impact angle. To investigate the effect of the impact angle, the particle mass per unit area of fiber is expressed as follows:

$$
w_{c}=\rho \cdot t / A \quad\left(\mathrm{~g} / \mathrm{m}^{2}\right)
$$

Where $t$ is wear time (sec), A is true contact area of the particle $\left(\mathrm{m}^{2}\right)$ as in Fig. 5, and $\rho$ is the particle supply rate by the micro feeder $(\mathrm{g} / \mathrm{sec})$.

Figure 6 illustrates the relationship between particle mass per unit area and breaking stress of the Procon fiber. The impact angles are 15, 30, 45, 60, 75 and 90 degrees. Other experiment conditions are the same as in Fig. 3 and 4. The cross axle is the particle mass per unit area $\left(W_{c}\right)$. As $W_{c}$ increases, the breaking stress of fiber decreases, and the decrease of breaking stress of fiber is related to the impact angle, as illustrated in Fig. 6. The decreased of fiber breaking stress at 30 and 40 degrees is significant, indicating sensitivity to erosion. The decrease at 90 degrees is the smallest, indicating resistance to erosion.

Fig. 7 illustrates the relationship between particle mass per unit area and breaking stress of the Kurabara $135 \mathrm{~d}$ fiber. The particle supply rate is $0.360 \mathrm{~g} / \mathrm{min}$, and the collision speed is $80.14 \mathrm{~m} / \mathrm{sec}$. Other experiment conditions are the same as for Procon. Figure 8 illustrates the relationship between particle mass per unit area and breaking stress of the DE-75 fiber. The particle supply rate is $0.516 \mathrm{~g} / \mathrm{min}$, and the collision speed is $46.34 \mathrm{~m} / \mathrm{sec}$. Other experiment conditions are the same as for Procon. In Fig. 8, the decrease of fiber breaking stress at 75 and 90 degrees is significant, indicating sensitivity to erosion. The decrease at 15 and 30 degrees is the smallest, indicating robustness to erosion.

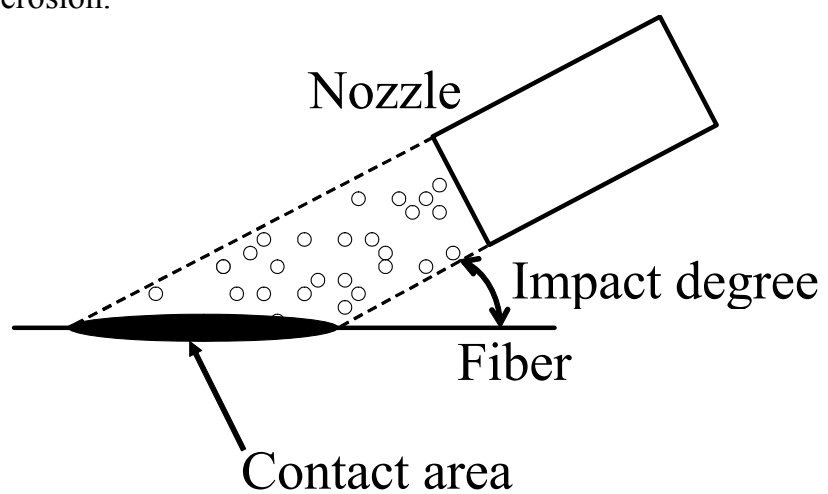

Fig. 5 True contact area of the particle

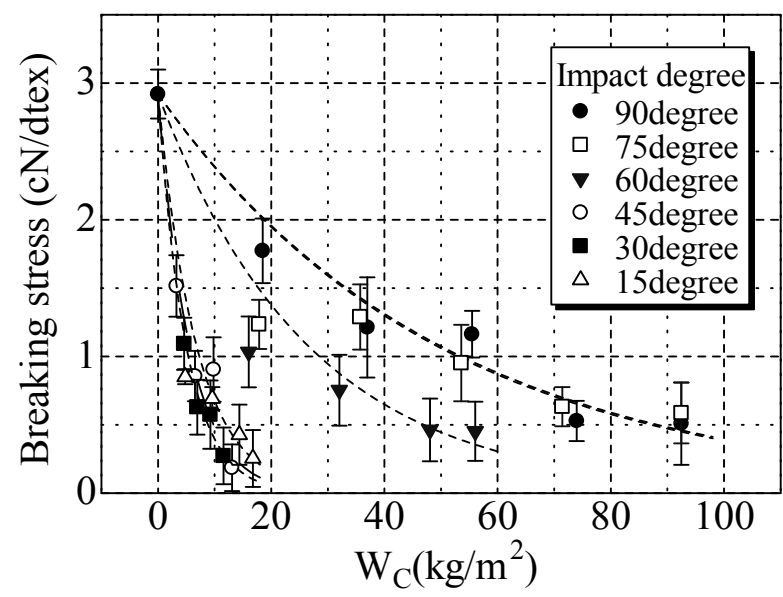

Fig.6 Particle mass- breaking stress curve for Procon

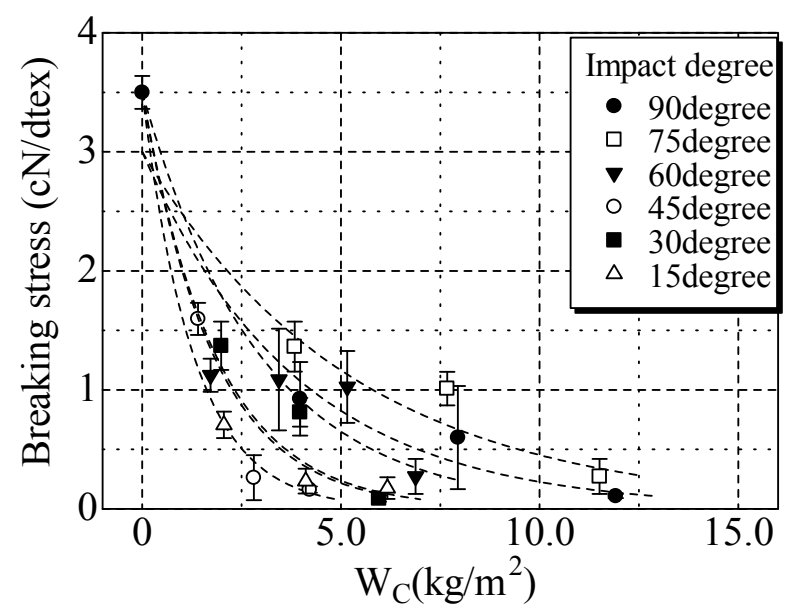

Fig.7 Particle mass- breaking stress curve for fibers (Kurebara135d)

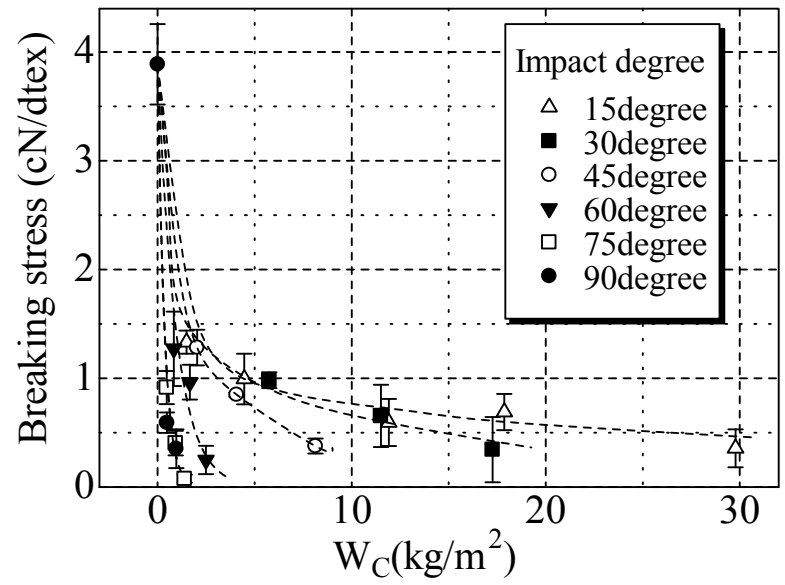

Fig.8 Particle mass- breaking stress curve for fibers (DE-75)

Fiber surface damage was observed with SEM. Figure 9 provides SEM photographs of fibers before and after erosion (a: Procon, b: Kurebara135d, c: DE-75). The impact angle is 45 degrees. Procon fiber is damaged in the direction collided by particles, as in Fig. 9(a). The surface of fiber is cut, and the ductility damage is apparent. The results were the same for Kurebara135d. However, some cracks are observed on the fiber surface with glass fiber 
(DE-75), and the brittle damage is evident in Fig. 9(c).

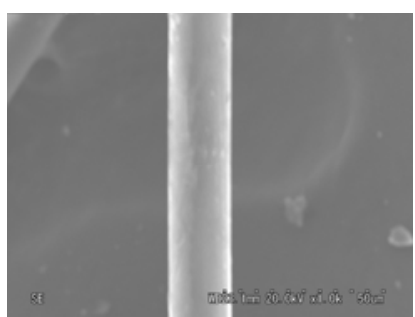

Before

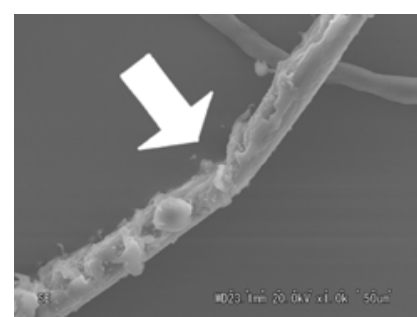

After (a) Procon

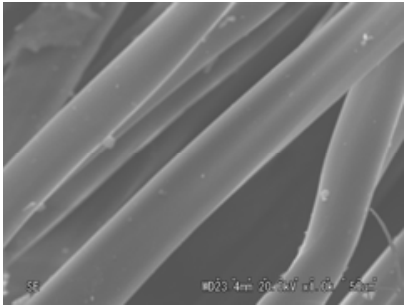

Before

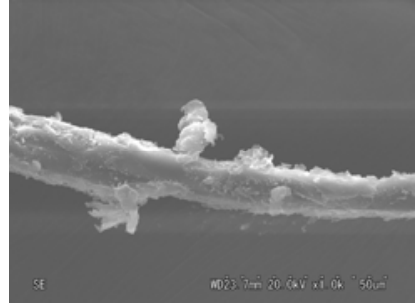

After (b) Kurebara135d

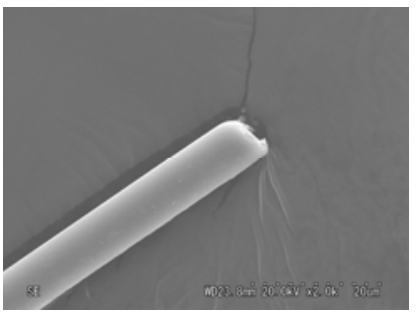

Before

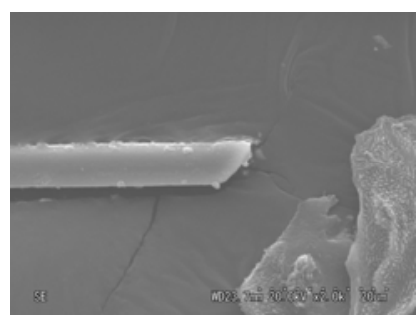

After (c) DE-75

Fig.9 Photographs of fibers (Impact angle 45 degrees)

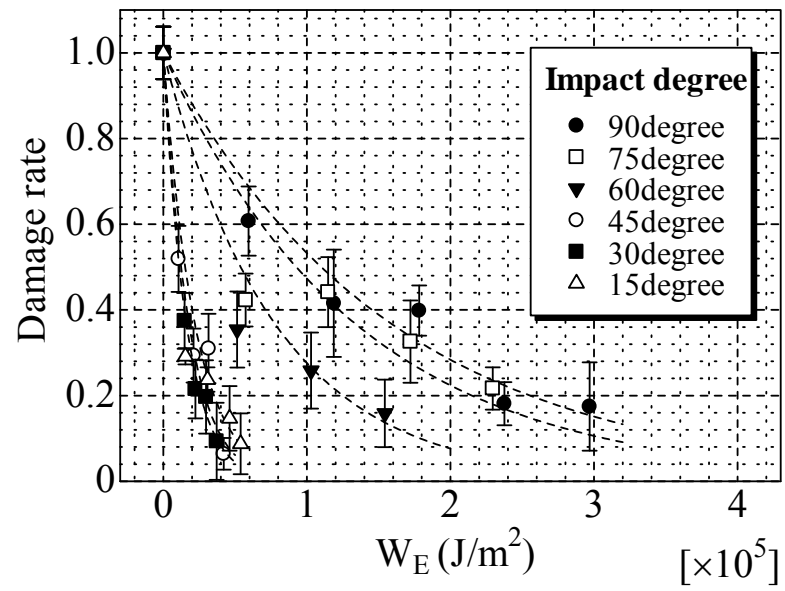

Fig.10 Kinetic energy of impact - Damage rate curve for fibers (Procon)

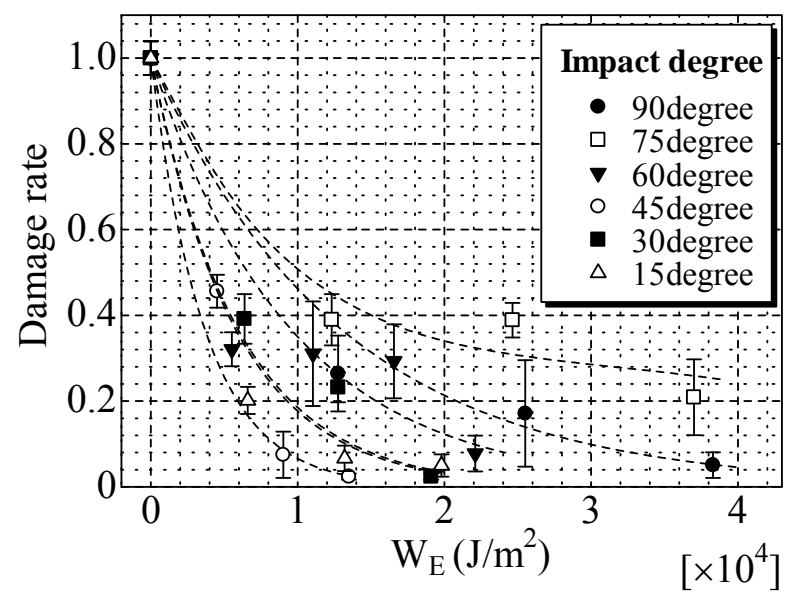

Fig.11 Kinetic energy of impact - Damage rate curve for fibers (Kurebara135d).

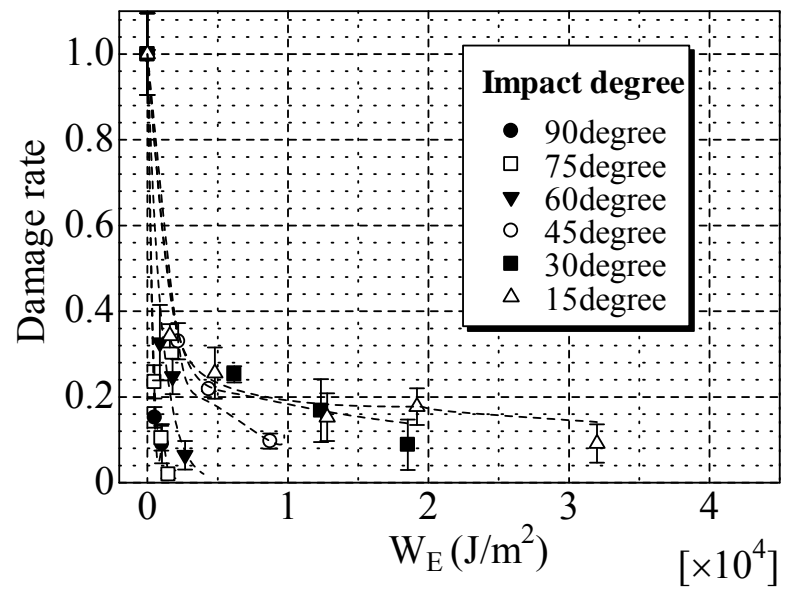

Fig.12 Kinetic energy of impact - Damage rate curve for fibers (DE-75).

The particle mass per unit area-breaking stress curve compares erosion characteristics of different impact angles for a fiber. However, we cannot compare the erosion characteristic of fibers at different impact speeds because the effect of impact speed is not included. We investigated the erosion characteristics of fibers using the sum of kinetic energy of particles before colliding with fibers (kinetic energy of impact: $W_{E}$ ) expressed as

$$
W_{E}=W_{c} V^{2} / 2 \times 10^{-3} \quad\left(\mathrm{~J} / \mathrm{m}^{2}\right)
$$

where $V$ is the impact speed $(\mathrm{m} / \mathrm{sec})$.

$W_{C}$ in cross axis of Fig. 6, 7 and 8 is changed like $W_{E}$. Fig. 10 to 12 illustrated the relationship between $W_{E}$ and damage rate of fibers. The damage rate is defined as:

$$
\text { Damage rate }=\frac{\text { Breaking stress }_{\text {after }}}{\text { Breaking stress }_{\text {before }}}
$$

Breaking stress ${ }_{\text {after }}$ is the breaking stress after fiber wear, and Breaking stress before is the breaking stress before fiber wear. Figure 10 illustrates the relationship between $W_{E}$ and the damage rate of the Procon fiber. The experiment conditions are the same as in Fig. 6. Figure 11 depicts the Kurebara135d fiber, and Fig. 12, the DE-75 fiber. Comparison of the erosion characteristics between fibers 
was possible using $W_{E}$.

The damage rate of Kurebara135d and DE-75 decreased more rapidly than that of Procon near 90 degrees, as the figures indicate. Our results demonstrate that the Procon fiber resists erosion wear the most. The Kurebara135d fiber is the next resistant, with the DE-75 fiber most vulnerable to erosion wear. The damage rate decreases more suddenly at 90 degrees than near 30 degrees and 45 degrees. However, Kurebara135d approach those of DE-75. Near 15 degrees, Kurebara135d and DE-75 fiber erosion characteristics reverse. Procon and Kurebara135d fiber displayed ductile material characteristics during erosion; DE-75 displayed brittle material characteristics.

Figure 13 illustrates the erosion mechanism of fibers. At the point of impact, the force on the fiber resolves into two components, $F_{N}=f \sin \theta$ and $F_{l}=f \cos \theta$, where $\vartheta$ is the angle of impact. Force $F_{N}$ compresses and impacts the fiber. Impacts can generate cracks in glass fibers composed of brittle materials. The SEM photograph in Fig. 9 is an example. PPS fibers of ductile materials are dented heavily and absorb the impact energy. Force $F_{l}$ generates a shear force that deforms the fiber. The erosion of ductile materials is greatly influenced by the shear strength.Therefore when solid particles impact with the brittle material, cracks appear on the material's surface and it becomes worn because of the collapse of cracks. The brittle material is vulnerable to erosion wear near 90 degrees. However, when solid particles impact the ductile material, the material surface became deeply dented, partially due to lifting and cutting by the impact force in cross direction, and it becomes worn. The ductile material is vulnerable to erosion wear near low angles. Materials like Procon fiber that have high strength and elongation are most resistant to erosion wear.
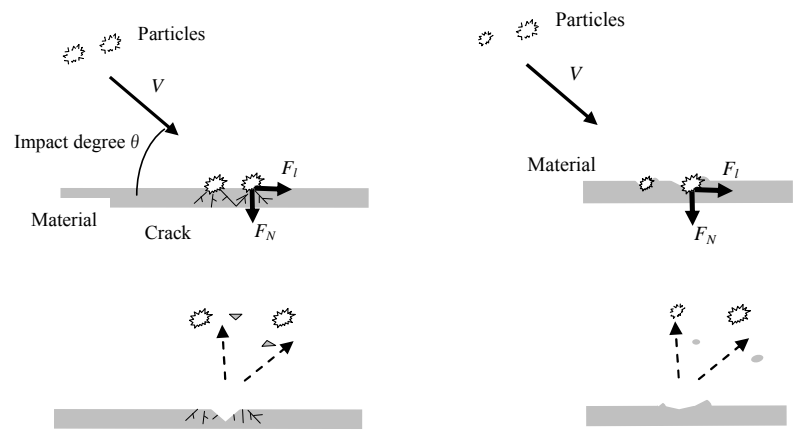

Brittle-material

Fig.13 The erosion mechanism of fiber

\section{CONCLUSIONS}

In this study, we developed an experiment and evaluation method to investigate the dry sand erosion characteristic of fiber by solid particle.

The conclusions were followed like the next:

1. The erosion wear of fiber depended on the kinetic energy of impact. It was feasible to use the kinetic energy as the evaluation value to evaluate the erosion wear under different experiment conditions.

2. The erosion wear levels of fibers were changed with the impact angle of solid particles. Furthermore, the degree of erosion damage was depends on the type of fiber used (brittle or ductile).

3. The Procon fiber showed higher erosion resistance than Kurebara135d and DE-75.It was considered that the erosion characteristics are significantly influenced by strength and elongation of materials.

\section{ACKNOWLEDGMENTS}

This work was supported by a Grant-in-Aid for Scientific Research (C)(2) 16560067 by the Ministry of Education, Culture, Sports, Science, and Technology of Japan.

\section{REFERENCES}

1. T. KISHIMA, L. BAO and K. NAKAZAWA, Experimental study about particle wear of the bag filters, Society of powder technology of JAPAN preprints, PP.55-57,(2003).

2. Schick M.J., Surface characteristics of fibers and textiles Part I, Marcel Dekker Inc, NY, 1975, PP.1-66.

3. Kenji Hashimoto, Wear Resistance Designing of Particulate, Powder Science and Engineering, P122-128, (1987).

4. Kouji Kimura and Heihatiro Okabe; Introduction to Etiology, YOKENDO Inc., 1982, P178-191.

5. K.V. Pool, C.K.H. Dharan, I. Finnie, Erosive wear of composite materials, Wear, 107, 1-12(1986).

6. A.P. Harsha, U.S. Tewari, B. Venkatraman, Solid particle erosion behaviour of various polyaryletherketone composites, Wear, 254, 693-712 (2003).

7. A. Häger, K. Friedrich, Y.A. Dzenis, S.A. Paipetis, Study of erosion wear of advanced polymer composites, in: K. Street, B.C. Whistler (Eds.), Proceedings of the ICCM-10, Canada Woodhead Publishing Ltd., Cambridge, 1995, PP. 155-162.

8. N.M. Barkoula, J. Karger-Kocsis, Effects of fibre content and relative fibre-orientation on the solid particle erosion of GF/PP composites, Wear, 252, 80-87(2002). 